\title{
Utilização de espécies exóticas na arborização e a facilitação para o estabelecimento de casos de invasão biológica
}

\author{
Juliano Ricardo Fabricante ${ }^{1}$ * \\ João Paulo Bispo Santos ${ }^{2}$ \\ Kelianne Carolina Tragino de Araújo ${ }^{3}$ \\ Vinicius Messas Cotarelli ${ }^{3}$ \\ ${ }^{1}$ Universidade Federal de Sergipe, Campus Universitário Prof. Alberto Carvalho \\ Departamento de Biociências, CEP 49500-000, Itabaiana - SE, Brasil \\ ${ }^{2}$ Universidade Federal de Sergipe, São Cristóvão - SE, Brasil \\ ${ }^{3}$ Universidade Federal do Vale do São Francisco, Petrolina - PE, Brasil \\ * Autor para correspondência \\ julianofabricante@hotmail.com
}

Submetido em 27/09/2016

Aceito para publicação em 16/01/2017

\section{Resumo}

O presente estudo teve como objetivo inventariar as espécies utilizadas na arborização do campus de Ciências Agrárias (CCA) da Universidade Federal da Paraíba (UFPB), Areia, PB e classificá-las de acordo com sua origem e seu potencial invasor. Por meio de caminhadas por toda a área de estudo (busca ativa) foram inventariadas todas as espécies utilizadas na arborização local e classificadas em nativas e exóticas. As exóticas ainda foram classificadas quanto ao seu potencial invasor. Ao todo foram identificadas 76 espécies pertencentes a 67 gêneros e 25 famílias. Destas, apenas 26 espécies eram nativas. Os resultados do presente trabalho são preocupantes devido ao elevado número de espécies exóticas utilizadas na arborização do local de estudo (50 spp.) e pela presença de espécies reconhecidamente agressivas, a exemplo de Artocarpus heterophyllus Lam., Azadirachta indica A. Juss. e Leucaena leucocephala (Lam.) de Wit.

Palavra-chave: Exóticas invasoras; Paisagismo

\section{Abstract}

Use of exotic species in afforestation and facilitation for the establishment of biological invasion. This study aimed to inventory the species used in landscaping the Campus of Agricultural Sciences of the Federal University of Paraíba, Areia, PB, Brazil and to rank them according to their origin and their invasive potential. Through walks throughout the study area (active search), we cataloged all the species used in local afforestation and classified them as native or exotic. Exotic plants were also classified as to their invasive potential. Altogether, we identified 76 species belonging to 67 genera and 25 families. Of these, only 26 species were native. The results of this study are worrisome because of the large number of exotic species used for planting at the study site (50 species), including known aggressive species: Artocarpus heterophyllus Lam., Azadirachta indica A. Juss. and Leucaena leucocephala (Lam.) de Wit.

Key words: Invasive exotic plants; Landscaping 


\section{Introdução}

No Brasil, a seleção de espécies para a arborização costuma ser feita segundo critérios estéticos (GONÇALVES et al., 2004) e sem levar em consideração a origem dos táxons (PIRES et al., 2008; 2010; SANTOS et al., 2008; PAIVA, 2009; BOHNER et al., 2011; KRAMER; KRUPEK, 2012; RICHTER et al., 2012; SCHALLENBERGER; MACHADO, 2013). Esse fato é extremamente preocupante uma vez que diversas dessas espécies passam a se dispersar para outros lugares, incluindo ambientes naturais, causando assim diferentes impactos sobre o meio (SAMPAIO; SCHMIDT, 2013). As espécies exóticas capazes de causarem esses efeitos são chamadas de exóticas invasoras e o processo é conhecido por invasão biológica (ESPÍNOLA; FERREIRA, 2007).

As invasões biológicas são consideradas a segunda maior causa em perda de biodiversidade do planeta, igualando-se aos efeitos das mudanças climáticas e perdendo apenas para a exploração direta de habitats (IUCN, 2000). Ademais, espécies exóticas invasoras, além de afetarem a biodiversidade, podem também influenciar a economia, a cultura e a saúde humana e animal (McNEELY et al., 2001).

Com isso, o presente estudo teve como objetivo inventariar as espécies utilizadas na arborização do campus de Ciências Agrárias (CCA) da Universidade Federal da Paraíba (UFPB), Areia, PB e classificá-las de acordo com sua origem e seu potencial invasor.

\section{Material e Métodos}

\section{Local de estudo}

O trabalho foi realizado no campus de Ciências Agrárias, UFPB, Areia, PB (658'1,9”'S e 3543'4,2”W; altitude média de $592 \mathrm{~m}$ ). O local apresenta uma área de aproximadamente 200 ha, sendo que 60 ha são compostos por fragmentos de Floresta Ombrófila Aberta (Brejo de altitude, disjunção de Mata Atlântica). O clima da região segundo a classificação de Köppen é do tipo As, ou seja, quente úmido (ALVARES et al., 2013). Os solos predominantes na região são os Podzólicos
Vermelho-Amarelo Equivalente Eutrófico (CAMPOS; QUEIROZ, 2006).

\section{Metodologia}

O inventário das espécies arbóreas foi feito através de caminhadas por todo o campus. As espécies inventariadas foram classificadas quanto ao seu status em nativas ou exóticas. Considerou-se como nativa (N) toda espécie com distribuição natural nas Florestas Ombrófilas Abertas, e exótica, toda espécie com origem diferente da anteriormente indicada. Para tanto foi consultada a origem das espécies na Lista de Espécies da Flora do Brasil (2016) e no Tropicos (2014). As exóticas ainda foram classificadas quanto ao seu potencial invasor em três grupos: EI - exóticas sem dispersão no campus; EII - exóticas com dispersão apenas em ambientes antrópicos e; EIII - exóticas com dispersão em todo o campus incluindo os fragmentos de Floresta Ombrófila Aberta (especialmente suas bordas). Essa categorização foi feita com base em observações realizadas durante a coleta de dados.

A identificação dos táxons foi realizada de acordo com literatura especializada e consulta de imagens de exsicatas disponibilizadas na Lista de Espécies da Flora do Brasil (2016), no Tropicos (2014) e na rede speciesLink (2016). A classificação taxonômica foi baseada no sistema APG III (2009), e a grafia dos nomes dos classificadores das espécies, na Lista de Espécies da Flora do Brasil (2016). Além disso, as espécies foram qualificadas segundo Saravy et al. (2003) quanto ao seu tipo de dispersão em: autocórica - dispersão feita pela própria planta; anemocórica - dispersão feita pelo vento; e zoocórica - dispersão feita pela fauna.

\section{Resultados}

Foram identificadas 76 espécies pertencentes a 67 gêneros e 25 famílias (Tabela 1). As famílias mais representativas foram Fabaceae (20), Anacardiaceae (7) e Bignoniaceae (6).

Entre as espécies amostradas, 26 (34,21\%) eram nativas e $50(65,78 \%)$ eram exóticas, das quais 10 $(13,15 \%)$ pertencem à categoria EI, $37(48,68 \%)$ à 
categoria EII e três $(3,94 \%)$ à categoria EIII. Em relação às origens, $13(17,10 \%)$ eram da América do Sul, 11 $(14,47 \%)$ da América Central, duas (2,63\%) da América do Norte, quatro $(5,26 \%)$ da África, duas $(2,63 \%)$ da Europa, 11 (14,47\%) da Ásia, uma (1,31\%) da Oceania, sete $(9,21 \%)$ com origem em mais de um continente, uma $(1,31 \%)$ pantropical e uma $(1,31 \%)$ das espécies era um híbrido. Além disso, de acordo com o tipo de dispersão, obtivemos que 36 (47,36\%) espécies eram zoocóricas, $21(27,63 \%)$ autocóricas e 19 (25\%) anemocóricas.

TABELA 1: Relação de espécies inventariadas do campus do CCA, UFPB, Areia, PB.

\begin{tabular}{|c|c|c|c|c|c|c|}
\hline Família & Nome científico & Nome vulgar & $\begin{array}{c}\text { Regiões de } \\
\text { ocorrência } \\
\text { natural }\end{array}$ & $\begin{array}{l}\text { Ocorrência } \\
\text { em FOA* }\end{array}$ & Dispersão & Categorias \\
\hline \multirow{7}{*}{ Anacardiaceae } & Anacardium occidentale L. & Cajú & Brasil & $\operatorname{Sim}$ & Zoocórica & $\mathrm{N}$ \\
\hline & $\begin{array}{l}\text { Myracrodruon urundeuva } \\
\text { Allemão }\end{array}$ & Aroeira & Brasil & Sim & Anemocórica & $\mathrm{N}$ \\
\hline & Mangifera indica L. & Mangueira & Ásia & Não & Zoocórica & EII \\
\hline & Schinopsis brasiliensis Engl. & Baraúna & Brasil & $\operatorname{Sim}$ & Anemocórica & $\mathrm{N}$ \\
\hline & Schinus terebinthifolius Raddi & Aroeira da praia & América do Sul & $\operatorname{Sim}$ & Zoocórica & $\mathrm{N}$ \\
\hline & Spondias mombin L. & Cajá & América & Não & Zoocórica & EI \\
\hline & Spondias sp. & Umbuguela & Hibrido & Não & Zoocórica & EI \\
\hline Annonaceae & Annona muricata L. & Graviola & Antilhas & Não & Zoocórica & EI \\
\hline Apocynaceae & $\begin{array}{l}\text { Tabernaemontana hystrix } \\
\text { Steud. }\end{array}$ & Leiteiro & Brasil & Sim & Zoocórica & $\mathrm{N}$ \\
\hline Araliaceae & $\begin{array}{l}\text { Schefflera morototoni (Aubl.) } \\
\text { Maguire, Steyerm. \& Frodin }\end{array}$ & Sambaquim & América do Sul & Sim & Zoocórica & $\mathrm{N}$ \\
\hline \multirow{5}{*}{ Arecaceae } & $\begin{array}{l}\text { Dypsis lutescens (H. Wendl.) } \\
\text { Beentje \& Dransf. }\end{array}$ & Palmeira areca & África & Não & Anemocórica & EII \\
\hline & Syagrus cearensis Noblck & Coco catolé & Brasil & $\operatorname{Sim}$ & Zoocórica & $\mathrm{N}$ \\
\hline & Caryota urens L. & Palmeira mulambo & Ásia & Não & Zoocórica & EI \\
\hline & Roystonea sp. & Palmeira imperial & América Central & Não & Zoocórica & EI \\
\hline & $\begin{array}{l}\text { Synechanthus warscewiczianus } \\
\text { H. Wendl. }\end{array}$ & Palmeira mexicana & $\begin{array}{c}\text { Panamá e Costa } \\
\text { Rica }\end{array}$ & Não & Zoocórica & EI \\
\hline \multirow{6}{*}{ Bignoniaceae } & Crescentia cujete L. & Cuité & América central & Não & Zoocórica & EI \\
\hline & $\begin{array}{l}\text { Spathodea campanulata } \\
\text { P. Beauv. }\end{array}$ & Spatodia & África & Não & Anemocórica & EI \\
\hline & $\begin{array}{l}\text { Handroanthus albus (Cham.) } \\
\text { Mattos }\end{array}$ & Ipê-branco & Brasil & Sim & Anemocórica & $\mathrm{N}$ \\
\hline & $\begin{array}{l}\text { Handroanthus chrysotrichus } \\
\text { (Mart. ex A. DC.) Mattos }\end{array}$ & Ipê-amarelo & Brasil & Não & Anemocórica & EII \\
\hline & $\begin{array}{l}\text { Handroanthus impetiginosus } \\
\text { Mattos }\end{array}$ & Ipê-roxo & Brasil & Sim & Anemocórica & $\mathrm{N}$ \\
\hline & $\begin{array}{l}\text { Tabebuia aurea (Silva Manso) } \\
\text { Benth. \& Hook. f. ex S. Moore }\end{array}$ & Caraibeira & Brasil & Sim & Anemocórica & $\mathrm{N}$ \\
\hline \multirow{2}{*}{ Bombacaceae } & $\begin{array}{l}\text { Ceiba glaziovii (Kuntze) K. } \\
\text { Schum. }\end{array}$ & Barriguda & Brasil & Sim & Anemocórica & $\mathrm{N}$ \\
\hline & Pachira aquatica Aubl. & $\begin{array}{l}\text { Castanha do } \\
\text { maranhão }\end{array}$ & Brasil & Não & Autocórica & EII \\
\hline Chrysobalanaceae & Licania rigida Benth. & Oiticica & América do Sul & Sim & Zoocórica & $\mathrm{N}$ \\
\hline Combretaceae & Terminalia catappa L. & Castanhola & África e Ásia & Não & Zoocórica & EII \\
\hline
\end{tabular}




\begin{tabular}{|c|c|c|c|c|c|c|}
\hline \multirow[b]{2}{*}{ Euphorbiaceae } & Cnidoscolus quercifolius Pohl & Faveleira & Brasil & Não & Autocórica & EI \\
\hline & $\begin{array}{l}\text { Euphorbia pulcherrima Willd. } \\
\text { ex Klotzsch }\end{array}$ & Bico de papagaio & América Central & Não & Autocórica & EI \\
\hline \multirow{20}{*}{ Fabaceae } & $\begin{array}{l}\text { Amburana cearensis } \\
\text { (Allemão) A.C. Sm. }\end{array}$ & Imburana de cheiro & Brasil & Sim & Anemocórica & $\mathrm{N}$ \\
\hline & $\begin{array}{l}\text { Anadenanthera colubrina } \\
\text { (Vell.) Brenan }\end{array}$ & Angico & Brasil & Sim & Autocórica & $\mathrm{N}$ \\
\hline & Bauhinia variegata $\mathrm{L}$. & Pata de vaca & Ásia & Não & Autocórica & EI \\
\hline & Brownea grandiceps Jacq. & $\begin{array}{l}\text { Rosa das } \\
\text { montanhas }\end{array}$ & Europa & Não & Autocórica & EI \\
\hline & $\begin{array}{l}\text { Paubrasilia echinata (Lam.) } \\
\text { Gagnon, H.C. Lima \& G.P. } \\
\text { Lewis }\end{array}$ & Pau-brasil & Brasil & Não & Autocórica & EII \\
\hline & $\begin{array}{l}\text { Poincianella pyramidalis } \\
\text { (Tul.) L.P. Queiroz }\end{array}$ & Catingueira & Brasil & Não & Autocórica & EI \\
\hline & Cassia grandis L. f. & Canafistula & Brasil & Não & Autocórica & EII \\
\hline & $\begin{array}{l}\text { Clitoria fairchildiana R.A. } \\
\text { Howard }\end{array}$ & Sombreiro & América do Sul & Não & Autocórica & EII \\
\hline & $\begin{array}{l}\text { Delonix regia (Boj. ex Hook.) } \\
\text { Raf. }\end{array}$ & Flamboyant & África & Não & Autocórica & EI \\
\hline & $\begin{array}{l}\text { Enterolobium contortisiliquum } \\
\text { (Vell.) Morong }\end{array}$ & Tamboril & Brasil & Sim & Autocórica & $\mathrm{N}$ \\
\hline & $\begin{array}{l}\text { Erythrina mulungu Mart. ex } \\
\text { Benth. }\end{array}$ & Mulungú & Brasil & Não & Autocórica & EI \\
\hline & $\begin{array}{l}\text { Gliricidia sepium (Jacq.) } \\
\text { Kunth ex Walp. }\end{array}$ & Gliricídia & $\begin{array}{l}\text { América do Sul e } \\
\text { América Central }\end{array}$ & Não & Autocórica & EI \\
\hline & Hymenaea courbaril L. & Jatobá & Brasil & Sim & Autocórica & $\mathrm{N}$ \\
\hline & Inga sp. & Ingá & América do Sul & Não & Zoocórica & EI \\
\hline & $\begin{array}{l}\text { Leucaena leucocephala (Lam.) } \\
\text { de Wit. }\end{array}$ & Leucena & América Central & Não & Autocórica & EIII \\
\hline & $\begin{array}{l}\text { Libidibia ferrea (Mart. ex Tul) } \\
\text { L.P. Queiroz }\end{array}$ & Pau-ferro & Brasil & Sim & Autocórica & $\mathrm{N}$ \\
\hline & Mimosa caesalpiniifolia Benth & Sabiá & Brasil & Não & Autocórica & EI \\
\hline & Pterodon emarginatus Vogel & Sucupira & Brasil & $\operatorname{Sim}$ & Anemocórica & $\mathrm{N}$ \\
\hline & $\begin{array}{l}\text { Pterodon pubescens (Benth.) } \\
\text { Benth. }\end{array}$ & Faveira & Brasil & Não & Anemocórica & EI \\
\hline & Pterogyne nitens Tul. & Madeira nova & Brasil & $\operatorname{Sim}$ & Anemocórica & $\mathrm{N}$ \\
\hline Lecythidaceae & Bertholletia excelsa Bonpl. & Castanha do Pará & Brasil & Não & Autocórica & EI \\
\hline \multirow[b]{2}{*}{ Malpighiaceae } & Lophanthera lactescens Ducke & Lanterneira & América do Sul & Não & Autocórica & EI \\
\hline & Malpighia glabra L. & Acerola & $\begin{array}{l}\text { América do Sul e } \\
\text { América Central }\end{array}$ & Não & Anemocórica & EI \\
\hline \multirow{3}{*}{ Meliaceae } & Azadirachta indica A. Juss. & Nim & Ásia & Não & Zoocórica & EIII \\
\hline & Cedrela fissilis Vell. & Cedro & Brasil & Não & Anemocórica & EI \\
\hline & Swietenia macrophylla King. & Mogno & Brasil & Não & Anemocórica & EI \\
\hline \multirow[t]{2}{*}{ Moraceae } & $\begin{array}{l}\text { Artocarpus altilis (Parkinson) } \\
\text { Fosberg }\end{array}$ & Fruta pão & Indonésia & Não & Zoocórica & EI \\
\hline & Artocarpus heterophyllus Lam. & Jaca & Ásia & Não & Zoocórica & EIII \\
\hline
\end{tabular}




\begin{tabular}{|c|c|c|c|c|c|c|}
\hline & $\begin{array}{l}\text { Ficus adhatodifolia Schott ex } \\
\text { Spreng. }\end{array}$ & Gameleira & Brasil & Sim & Zoocórica & $\mathrm{N}$ \\
\hline & Ficus benjamina $\mathrm{L}$. & Ficus & Ásia & Não & Zoocórica & EI \\
\hline \multirow{4}{*}{ Myrtaceae } & Eucalyptus citriodora Hook. & Eucaliptus & Oceania & Não & Anemocórica & EI \\
\hline & Eugenia jambos L. & Jambeiro & $\begin{array}{l}\text { Indonésia e } \\
\text { Malásia }\end{array}$ & Não & Zoocórica & EI \\
\hline & Eugenia uniflora $\mathrm{L}$. & Pitanga & América do Sul & Não & Zoocórica & EI \\
\hline & Psidium guajava $\mathrm{L}$. & Goiabeira & $\begin{array}{l}\text { América do Sul e } \\
\text { América Central }\end{array}$ & Não & Zoocórica & EII \\
\hline Oleaceae & Olea europaea $\mathrm{L}$. & Oliveira & Europa & Não & Zoocórica & EI \\
\hline Pinaceae & Pinus caribaea Morelet & Pinus & $\begin{array}{l}\text { América Central } \\
\text { e América do } \\
\text { Norte }\end{array}$ & Não & Anemocórica & EII \\
\hline Rhamnaceae & Ziziphus joazeiro Mart. & Juá & Brasil & Não & Zoocórica & EI \\
\hline \multirow{2}{*}{ Rubiaceae } & $\begin{array}{l}\text { Calycophyllum spruceanum } \\
\text { (Benth.) K. Schum. }\end{array}$ & Pau-mulato & Brasil & Sim & Anemocórica & $\mathrm{N}$ \\
\hline & Genipa americana $\mathrm{L}$. & Jenipapo & $\begin{array}{l}\text { América do Sul e } \\
\text { América Central }\end{array}$ & Sim & Zoocórica & $\mathrm{N}$ \\
\hline \multirow[b]{2}{*}{ Sapindaceae } & Litchi chinensis Sonn. & Lichieira & Ásia & Não & Zoocórica & EI \\
\hline & $\begin{array}{l}\text { Talisia esculenta (A. St.-Hil.) } \\
\text { Radlk }\end{array}$ & Pitomba & América do Sul & Não & Zoocórica & EI \\
\hline \multirow{3}{*}{ Sapotaceae } & Chrysophyllum cainito L. & Caimito & América Central & Não & Zoocórica & EI \\
\hline & $\begin{array}{l}\text { Manilkara inundata (Ducke) } \\
\text { Ducke }\end{array}$ & Abrico do mato & Brasil & Sim & Zoocórica & $\mathrm{N}$ \\
\hline & $\begin{array}{l}\text { Sideroxylon obtusifolium } \\
\text { (Humb. ex Roem. \& Schult) } \\
\text { T.D. Penn. }\end{array}$ & Quixabeira & Brasil & Não & Zoocórica & EI \\
\hline \multirow{2}{*}{ Simaroubaceae } & Simarouba amara Aubl. & Pau-paraíba & Brasil & Sim & Zoocórica & $\mathrm{N}$ \\
\hline & Simarouba $\quad$ sp. & Simaruba & Brasil & Sim & Zoocórica & $\mathrm{N}$ \\
\hline Sterculiaceae & Theobroma cacao L. & Cacau & América do Sul & Não & Zoocórica & EI \\
\hline Symplocaceae & $\begin{array}{l}\text { Symplocos uniflora (Pohl) } \\
\text { Benth. }\end{array}$ & Pau de cangalho & América do Sul & Sim & Zoocórica & $\mathrm{N}$ \\
\hline Verbenaceae & Tectona grandis L. f. & Teca & Ásia & Não & Autocórica & EI \\
\hline
\end{tabular}

*FOA = Floresta Ombrófila Aberta.

\section{Discussão}

Os resultados obtidos demonstram a predominância de espécies exóticas na arborização do local estudado. Blum et al. (2008) e Ziller et al. (2007) realizaram estudos semelhantes com registros de táxons análogos ao deste trabalho, porém, em regiões distintas, demonstrando que esse é um problema que se repete em todo o país.

As espécies Artocarpus heterophyllus Lam., Leucaena leucocephala (Lam.) de Wit. e Azadirachta indica A. Juss se enquadradam na categoria EIII e representam 3,94\% do total dos táxons amostrados. Apesar da baixa porcentagem, os resultados são extremamente preocupantes, uma vez que se trata de exóticas capazes de se dispersar em áreas de interesse conservacionista e são reconhecidamente agressivas (WILLIAMSON, 1999; MACK et al., 2000; PIRES et al., 2001; LEÃO et al., 2011a; PIMENTEL, 2011; FABRICANTE et al., 2012; FABRICANTE, 2014a)

Artocarpus heterophyllus Lam. - A jaqueira é uma espécie arbórea da família Moraceae, nativa da Ásia, introduzida no Brasil durante o período colonial 
(FERRÃO, 2004). A jaqueira é encontrada em quase todo território brasileiro com registros de invasão biológica em fragmentos de Mata Atlântica de diferentes partes do país (RODRIGUES, 2004; ABREU; RODRIGUES, 2005; CRUZ, 2007; FABRICANTE et al., 2012). Cruz (2007) verificou que áreas invadidas por A. heterophyllus possuem uma diversidade menor de espécies quando comparadas a áreas não invadidas. Além disso, em um estudo realizado por Fabricante et al. (2012), em um fragmento de Floresta Ombrófila Aberta no campus de Ciências Agrárias, UFPB, Areia, PB, foi constatado que $A$. heterophyllus tem a capacidade de alterar a composição, riqueza e diversidade arbórea autóctone assim como a química dos solos dos sítios invadidos. Ainda há outros trabalhos com a espécie, a exemplo do estudo de Boni et al. (2009), que avaliaram o início do processo de invasão em uma reserva biológica na Mata Atlântica do estado do Espírito Santo, e de Perdomo e Magalhães (2007), que aferiram os efeitos alelopáticos da A. heterophyllus sobre plantas adjacentes a ela. Desta forma, existe a necessidade e urgência de ações de manejo e controle da espécie em fragmentos de Mata Atlântica, principalmente daquelas que estão inseridas nos biomas brasileiros considerados como prioritários para a conservação da biodiversidade (hotspots).

Leucaena leucocephala (Lam.) de Wit. - A leucena é uma leguminosa da família Fabaceae, nativa do México, e bem adaptada às condições climáticas do Brasil (KILL; MENEZES, 2005). A espécie é muito utilizada em recuperação de áreas degradadas e empregada como alimento para animais (WEE; WANG, 1987). Devido a isso e por se dispersar eficientemente e se estabelecer em solos com baixa fertilidade (NOBLE, 1989), L. leucocephala foi bastante difundida pelo país. Essa espécie produz grande quantidade de sementes viáveis e é capaz de formar banco de sementes no solo com viabilidade entre 10 e 20 anos além de regenerarse rapidamente após corte ou queima (FONSECA; JACOBI, 2011). Como consequência, ela consegue formar extensos maciços populacionais, diminuindo o poder de resiliência das áreas e alterando dessa forma todos os processos ecossistêmicos naturais (YOSHIDA; OKA, 2004). Além disso, essa espécie também é hospedeira de diversas pragas de lavoura (ALVES et al., 2014). Em razão desse conjunto de fatores, a invasão dessa espécie é cada vez mais comum, e a espécie pode ser observada invadindo diferentes ecossistemas (HOROWITZ et al., 2013; MELLO, 2014; FABRICANTE et al., 2015).

Azadirachta indica A. Juss. - O nim ou neem foi introduzido no Brasil na década de 1940, possui hábito arbóreo e pertence à família Meliaceae (FABRICANTE, 2014b). Devido à sua propriedade bioinseticida (SCHMUTTERER, 1990) e ao seu rápido crescimento e copa frondosa (NEVES et al., 2013), a espécie foi disseminada em todo o país tanto na zona rural como nas cidades. Essa espécie é reconhecidamente alelopática (RICKLI et al., 2011; MORO et al., 2013), pois libera fitoquímicos (azadiractina) no solo impedindo a germinação e desenvolvimento das plantas ao seu redor (ASHRAFI et al., 2008), comprometendo assim a capacidade de resiliência dos ambientes invadidos (FABRICANTE, 2014a). A. indica também provoca a morte de vários polinizadores, especialmente das abelhas (ALVES, 2010).

Algumas espécies enquadradas na categoria EII merecem destaque, visto que são táxons com alta capacidade de dispersão e já são relatadas como exóticas invasoras em outros estudos, a exemplo de Pinus caribaea Morelet, Psidium guajava L. e Terminalia catappa L.

Pinus caribaea Morelet. - O pinheiro, como é popularmente chamado, é nativo da América do Norte e América Central, possui porte arbóreo e pertence à família Pinaceae (RIZZINI; MORS, 1995). A invasão biológica ocasionada por qualquer indivíduo desse gênero acarreta sérios problemas ambientais. As espécies do gênero Pinus são comumente encontradas em áreas campestres ou nas bordas das florestas, pois são heliófilas (VENZKE et al., 2012). Sua invasão causa inúmeras modificações no ambiente, como acidificação dos solos e consumo exacerbado de água, que às vezes chega a prejudicar o lençol freático, o que causa mudanças na composição das comunidades de plantas (LEÃO et al., 2011b). Na Colômbia, árvores desse gênero foram plantadas próximas à região andina e sua invasão acarretou a exclusão de espécies nativas (ZALBA, 2006). 
Psidium guajava L. - Chamada vulgarmente de goiabeira, $P$. guajava é representante da família Myrtaceae e nativa da América do Norte (MORTON, 1987). A espécie por muito tempo foi difundida como alternativa para recuperação de pastagens abandonadas (BERENS et al., 2008), além de ser uma frutífera amplamente cultivada no Brasil (PEREIRA; ANDRADE, 1994). Psidium guajava produz fitotoxinas (BAIS et al., 2003) que atingem principalmente o sistema radicular e a germinação de sementes de outras plantas (CHAPLA; CAMPOS, 2010). Santana e Encinas (2011) inventariaram essa espécie em áreas antropizadas coberta por vegetação em Brasília e Goiânia e Zviejkovski et al. (2009) observaram a ocorrência dessa espécie em uma Unidade de Conservação no Paraná.

Terminalia catappa L. - Conhecida popularmente como amendoeira, chapéu-de-sol ou sete-copas, $T$. catappa L. é nativa da Ásia (SANCHES et al., 2007) e pertence à família Combretaceae. Atualmente é encontrada em diversos países tropicais sendo utilizada especialmente na arborização de áreas urbanas devido ao sombreamento proporcionado por suas grandes copas (SANTOS et al., 2015). Há inúmeras hipóteses de como a espécie tenha chegado ao Brasil. Uma delas é de que suas sementes tenham vindo na água de lastro de navios (PLUCÊNIO et al., 2013). Os frutos de $T$. catappa possuem pericarpos espessos, o que os tornam atrativos para animais, que acabam dispersando (THOMSON; EVANS, 2006). Terminalia catappa não demanda recursos especiais para sua germinação (SANCHES et al., 2007) e é capaz de modificar a composição de espécies das áreas invadidas devido ao sombreamento promovido por sua copa e pela liberação de substâncias alelopáticas no ambiente (BARATELLI, 2006; SANCHES, 2009; COSTA, 2013).

Os resultados do presente trabalho são preocupantes devido ao elevado número de espécies exóticas utilizadas na arborização do local de estudo (50 spp.) e à presença de espécies reconhecidamente agressivas, a exemplo de Artocarpus heterophyllus Lam., Azadirachta indica A. Juss. e Leucaena leucocephala (Lam.) de Wit.

Ressalta-se que a utilização de espécies exóticas na arborização pode favorecer o início de processos de invasão biológica e gerar sérios impactos ambientais, reduzindo a vegetação nativa nas áreas invadidas. Sugere-se a substituição das exóticas por espécies nativas, que, além de desempenharem as mesmas funções paisagísticas e de conforto ambiental, ainda possuem relações harmônicas com outras espécies autóctones locais.

\section{Referências}

ABREU, R. C. R.; RODRIGUES, P. J. F. P. Estrutura de populações de jaqueiras, subsídios para manejo e conservação da Mata Atlântica. In: SIMPÓSIO BRASILEIRO SOBRE EXÓTICAS INVASORAS, I, 2005, Brasília. Anais... Brasília: FUNDEP, 2005. 14 p.

ALVARES, C.A.; STAPE, J.L.; SENTELHAS, P.C.; GONÇALVES, J. L. M.; SPAROVEK, G. Köppen's climate classification map for Brazil. Meteorologische Zeitschrift, Vienna, v. 22, n. 6, p. 711$728,2013$.

ALVES, J. E. Toxicidade do nim (Azadirachta indica A. Juss.: Meliaceae) para Apis mellifera e sua importância apícola na Caatinga e Mata Litorânea cearense. 2010. 142 f. Tese (Doutorado em Zootecnia) - Universidade Federal do Ceará, Fortaleza. 2010.

ALVES, J. S.; REIS, L. B. O.; SILVA, E. K. C.; FABRICANTTE, J. R.; FILHO, J. A. S. Leucaena leucocephala. In: FABRICANTE, J. R. (Ed.). Plantas exóticas e exóticas invasoras da Caatinga. Florianópolis: Bookess, 2014. p. 15-22.

APG III. An update of the Angiosperm Phylogeny Group classification for the orders and families of flowering plants: APG III. Botanical Journal of the Linnean Society, London, v. 161, p. 105-121, 2009.

ASHRAFI, Z. Y.; RAHNAVARD, A.; SADEGHI, S.; ALIZADE, H. M.; MASHHADI, R. Study of the allelopathic potential of extracts of Azadirachta indica (neem). International Journal of Biological Sciences, Cheongju City, v. 8, n. 3, p. 56-61, 2008.

BAIS, H. P.; VEPACHEDU, R.; GILROY, S.; CALLAWAY, R. M.; VIVANCO, J. M. Allelopathy and exotic plant invasion: from molecules and genes to species interactions. Science, Washington, v. 301, n. 5638, p. 1377-1380, 2003.

BARATELLI, T. G. Estudo das propriedades alelopáticas vegetais: investigação de substâncias aleloquímicas em Terminalia catappa L. (Combretaceae). 2006. 206 f. Tese (Doutorado em Química de Produtos Naturais) - Universidade Federal do Rio de Janeiro, Rio de Janeiro. 2006.

BERENS, D. G.; FARWING, N.; SCHAAB, G.; GAESEBOHNING, K. Exotic guavas are foci of forest regeneration in Kenyan farmland. Biotropica, Malden, v. 40, n. 1, p. 104-112, 2008. BLUM, C. T.; BORGO, M.; SAMPAIO, A. C. F. Espécies exóticas invasoras na arborização de vias públicas de Maringá - PR. Revista Sociedade Brasileira de Urbanização, Piracicaba, v. 3, n. 2, p. 78-97, 2008.

BOHNER, T.; GRACIOLI, C. R.; REDIN, C. G. Análise qualiquantitativa da arborização do município de Guatambu, SC. Revista Monografias Ambientais, Santa Maria, v. 3, n. 3, p. 532-546, 2011. BONI, R.; NOVELLI, F. Z.; SILVA, A. G. Um alerta para os riscos de bioinvasão de jaqueiras, Artocarpus heterophyllus Lam., na 
Reserva Biológica Paulo Fraga Rodrigues, antiga Reserva Biológica Duas Bocas, no Espírito Santo, sudeste do Brasil. Natureza Online, Santa Teresa, v. 7, n. 1, p. 51-55, 2009.

CAMPOS, M. C. C.; QUEIROZ, S. B. Reclassificação dos perfis descritos no Levantamento Exploratório-Reconhecimento de solos do estado da Paraíba. Revista de Biologia e Ciências da Terra, São Cristóvão, v. 6, n. 1, p. 45-50, 2006.

CHAPLA, T. E.; CAMPOS, J. B. Allelopathic evidence in exotic guava (Psidium guajava L.). Brazilian archives of Biology and Technology, Curitiba, v. 53, n. 6, p. 1359-1362, 2010.

COSTA, M. A. A. Influência da população da espécie exótica Terminalia catappa L. (Amendoeira) sobre espécies nativas numa área da Restinga, domínio Tropical Atlântico, Salvador, Bahia. 2013. 56 f. Dissertação (Mestrado em Ecologia e Biomonitoramento) - Universidade Federal da Bahia, Salvador. 2013.

CRUZ, A. R. Parque Natural Municipal Fazenda Atalaia, Macaé. 2007. 38 f. Monografia (Curso de Engenharia Florestal) - Universidade Federal Rural do Rio de Janeiro, Seropédica. 2007.

ESPÍNOLA, L. A.; FERREIRA, H. Espécies invasoras: conceitos, modelos e atributos. Interciencia, Caracas, v. 32, n. 9, p. 580-585, 2007.

FABRICANTE, J. R. Azadirachta indica. In: FABRICANTE, J. R.Plantas exóticas e exóticas invasoras da Caatinga. Florianópolis: Bookess, 2014b. p. 23-30.

FABRICANTE, J. R. Plantas exóticas e exóticas invasoras da Caatinga. Florianópolis: Bookess, 2014a. 50 p.

FABRICANTE, J. R.; ARAÚJO, K. C. T. A.; ANDRADE, L. A. A.; FERREIRA, J. V. A. F. Invasão biológica de Artocarpus heterophyllus Lam. (Moraceae) em um fragmento de Mata Atlântica no Nordeste do Brasil: impactos sobre a fitodiversidade e os solos dos sítios invadidos. Acta Botanica Brasilica, São Paulo, v. 26, n. 2, p. 399-407, 2012.

FABRICANTE, J. R.; ZILLER, S. R.; ARAÚJO, K. C. T.; FURTADO, M. D. G.; BASSO, F. A. Non-native and invasive alien plants on fluvial islands in the São Francisco River, northeastern Brazil. Check List, Rio Claro, v. 11, n. 1, p.1535-1546, 2015.

FERRÃO, J. E. M. A aventura das plantas e os descobrimentos portugueses. Lisboa: Instituto de Investigação Científica Tropical, 2004. 287 p.

FONSECA, N. G.; JACOBI, C. M. Desempenho germinativo da invasora Leucaena leucocephala (Lam.) de Wit. e comparação com Caesalpinia ferrea Mart. ex Tul. e Caesalpinia pulcherrima (L.) Sw. (Fabaceae). Acta Botanica Brasilica, São Paulo, v. 25, n. 1, p. 191-197, 2011.

GONÇALVES, E. O.; PAIVA, H. N.; GONÇALVES, W.; JACOVINE, L. A. G. Avaliação qualitativa de mudas destinadas à arborização urbana no estado de Minas Gerais. Revista Árvore, Viçosa, v. 28, n. 4, p. 479-486, 2004.

HOROWITZ, C.; MARTINS, C. R.; WALTER, B. M. T. Flora exótica no Parque Nacional de Brasília: Levantamento e classificação das espécies. Biodiversidade Brasileira, Brasília, v. 3, n. 2, p. 50-73, 2013.

IUCN - INTERNATIONAL UNION FOR CONSERVATION OF NATURE AND NATURAL RESOURCES. IUCN guidelines for the prevention of biodiversity loss caused by alien invasive species. Gland: IUCN, 2000. 25 p.
KILL, L. H. P.; MENEZES, E. A. Espécies vegetais exóticas com potencialidades para o semi árido brasileiro. Brasília: EMBRAPA Semi-Árido, 2005. 340 p.

KRAMER, J. A.; KRUPEK, R. A. Caracterização florística e ecológica da arborização de praças públicas do município de Guarapuava, PR. Revista Árvore, Viçosa, v. 36, n. 4, p. 647-658, 2012.

LEÃO, T. C. C.; ALMEIDA, W. R.; DECHOUM, M. S.; ZILLER, S. R. O problema das espécies exóticas invasoras. In: LEÃO, T. C. C.; ALMEIDA, W. R.; DECHOUM, M. S.; ZILLER, S. R. (Ed.). Espécies exótica invasoras no nordeste do Brasil: Contextualização, Manejo e Políticas Públicas. Recife: Cepan, 2011a. p. 9-14.

LEÃO, T. C. C.; ALMEIDA, W. R.; DECHOUM, M. S.; ZILLER, S. R. Plantas exóticas invasoras. In: LEÃO, T. C. C.; ALMEIDA, W. R.; DECHOUM, M. S.; ZILLER, S. R. (Ed.). Espécies exótica invasoras no nordeste do Brasil: Contextualização, Manejo e Políticas Públicas. Recife: Cepan, 2011b. p. 52-76.

LISTA DE ESPÉCIES DA FLORA DO BRASIL. Jardim Botânico do Rio de Janeiro. 2016. Disponível em: <www.floradobrasil.jbrj. gov.br/>. Acesso em: 23 julho 2016.

MACK, R. N.; SIMBERLOFF, P. D.; LONSDALE, W. M.; EVANS, H.; CLOUT, M.; BAZZAZ, F. Biotic invasions: causes, epidemiology, global consequences, and control. Ecological applications, Washington, v. 10, n. 3, p. 689-710, 2000.

McNEELY, J. A.; MOONEY, H. A.; NEVILLE, L. E.; SCHEI, P. J.; WAAGE, J. K. Global strategy on invasive alien species. Cambridge: IUCN, 2001. 62 p.

MELLO, T. J. Invasão biológica em ilhas oceânicas: o caso de Leucaena leucocephala (Leguminosae) em Fernando de Noronha. 2014. 96 f. Tese (Mestrado em Ciências) - Universidade de São Paulo, São Paulo. 2014.

MORO, M. F. WESTERKAMP, C.; MARTINS, F. R. Naturalization and potential impact of the exotic tree Azadirachta indica A. Juss. in Northeastern Brazil. Check List, Rio Claro, v. 9, n. 1, p. 153156, 2013.

MORTON, J. F. Fruits of warm climates. Winterville: Creative Resource Systems, 1987. 2997 p.

NEVES, E. J. M.; REISSMANN, C. B.; DEDECEK, R. A.; CARPANEZZI, A. A. Caracterização nutricional do nim em plantios no Brasil. Revista Brasileira de Engenharia Agrícola e Ambiental, Campina Grande, v. 17, n. 1, p. 26-32, 2013.

NOBLE, I. R. Attributes of invaders and the invading process: terrestrial and vascular plants. In: DRAKE, J. A.; DICASTRI, F.; GROVES, R. H.; KRUGER, F. J.; MOONEY, H. A.; REJMÁNEK, M.; WILLIAMSON, M. H. (Ed.). Biological invasions: a global perspective. New York: Willey, 1989. p. 301-313.

PAIVA, A. V. Aspectos da arborização urbana do Centro de Cosmópolis - SP. Revista da Sociedade Brasileira de Arborização Urbana, Piracicaba, v. 4, n. 4, p. 17-31, 2009.

PERDOMO, M.; MAGALHÃES, L. M. S. Ação alelopática da jaqueira (Artocarpus heterophyllus) em laboratório. Revista Floresta e Ambiente, Seropédica, v. 14, n. 1, p. 52-55, 2007.

PEREIRA, T. S.; ANDRADE, A. C. S. Germinação de Psidium guajava L. e Passiflora edulis Sims: efeito da temperatura, substrato e morfologia do desenvolvimento pós-seminal. Revista Brasileira de Sementes, Londrina, v. 16, n. 1, p. 58-62, 1994. 
PIMENTEL, D. (Ed.). Biological invasions: economic and environmental costs of alien plant, animal, and microbe species. Boca Raton: CRC Press, 2011. 384 p.

PIRES, N. A. M. T.; MELO, M. S. M.; OLIVEIRA, D. E.; XAVIERSANTOS, S. Diagnóstico da arborização urbana do município de Goiandira, Goiás. Revista Brasileira de Biociências, Porto Alegre, v. 5, n. S1, p. 537-539, 2008.

PIRES, N. A. M. T.; MELO, M. S. M.; OLIVEIRA, D. E.; XAVIERSANTOS, S. A arborização urbana do município de Goiandira/GO - caracterização qualiquantitativa e proposta de manejo. Revista da Sociedade Brasileira de Arborização Urbana, Piracicaba, v. 5, n. 3, p. 185-205, 2010.

PIRES, N. M.; SOUZA, I. R. P.; PRATES, H. T.; FARIA, T. C. L. F.; FILHO, I. A. P.; MAGALHÃES, P. C. Efeito do extrato aquoso de leucena sobre o desenvolvimento, índice mitótico e atividade da peroxidase em plântulas de milho. Revista Brasileira de Fisiologia Vegetal, Campinas, v. 13, n. 1, p. 55-65, 2001.

PLUCÊNIO, R. M.; DECHOUM, M. S.; CASTELLANI, T. T. Invasão biológica em restinga: o estudo de caso de Terminalia catappa L. (Combretaceae). Biodiversidade Brasileira, Brasília, v. 3, n. 2, p. 118-136, 2013.

RICHTER, C.; PEITER, M. X.; ROBAINA, A. D.; SOUZA, A. R. C. S.; FERRAZ, R. C.; DAVID, A. F. Levantamento da arborização urbana pública de Mata/RS. Revista da Sociedade Brasileira de Arborização Urbana, Piracicaba, v. 7, n. 3, p. 88-96, 2012.

RICKLI, H. C.; FORTES, A. M. T.; SILVA, P. S. S.; PILATTI, D. M.; HUTT, D. R. Efeito alelopático de extrato aquoso de folhas de Azadirachta indica A. Juss. em alface, soja, milho, feijão e picãopreto. Semina: Ciências Agrárias, Londrina, v. 32, n. 2, p. 473484, 2011.

RIZZINI, C. T.; MORS, W. B. Botânica econômica brasileira. Rio de Janeiro: Âmbito Cultural, 1995. 241 p.

RODRIGUES, P. J. F. P. A vegetação da Reserva Biológica União e os efeitos de borda na Mata Atlântica fragmentada. 2004. 136 f. Tese (Doutorado em Biociências e Biotecnologia) Universidade Estadual do Norte Fluminense, Rio de Janeiro. 2004.

SAMPAIO, A. B.; SCHMIDT, I. B. Espécies exóticas invasoras em unidades de conservação federais do Brasil. Biodiversidade Brasileira, Brasília, v. 3, n. 2, 32-49, 2013.

SANCHES, J. H. Potencial invasor do chapéu do Sol (Terminalia catappa L.) em área de restinga. 2009. 84 f. Dissertação (Mestrado em Recursos Florestais) - Universidade de São Paulo, Piracicaba. 2009.

SANCHES, J. H.; MAGRO, T. C.; DA SILVA, D. F. Distribuição espacial da Terminalia catappa L. em área de restinga no parque Estadual da Serra do Mar, Núcleo Picinguaba, Ubatuba/SP. In: SIMPÓSIO BRASILEIRO DE SENSORIAMENTO REMOTO, XIII, 2007, Florianópolis. Anais... Florianópolis, INPE, 2007. p. 1831-1838.

SANTANA, O. A.; ENCINAS, J. I. Levantamento das espécies exóticas arbóreas e seu impacto nas espécies nativas em áreas adjacentes a depósitos de resíduos domiciliares. Biotemas, Florianópolis, v. 21, n. 4, p. 29-38, 2011.

SANTOS, A. R.; BERGALlO, H. G.; ROCHA, C. F. D. Paisagem urbana alienígena. Revista Ciência Hoje, Rio de Janeiro, v. 41, n. 245, p. 68-73, 2008.
SANTOS, E. L.; SOUZA, A. P. L.; PONTES, E. C.; GONZAGA, L. S.; FERREIRA, A. J. S. Folha de amendoeira (Terminalia catappa) como aditivo promotor de crescimento em rações para alevinos de Tilápia do Nilo (Oreochromis niloticus). Agropecuária Técnica, Areia, v. 36, n. 1, p. 190-196, 2015.

SARAVY, F. P.; DE FREITAS, P. J.; LAGE, M. A.; LEITE, S. J.; BRAGA, L. F.; SOUSA, M. P. Síndrome de dispersão em estratos arbóreos em um fragmento de floresta ombrófila aberta e densa em Alta Floresta - MT. Revista do Programa de Ciências Agroambientais, Alta Floresta, v. 2, n. 1, p. 1-12, 2003.

SCHALLENBERGER, L. S.; MACHADO, G. O. Inventário da arborização na região central do município de MangueirinhaPR. Revista da Sociedade Brasileira de Arborização Urbana, Piracicaba, v. 8, n. 1, p. 54-64, 2013.

SCHMUTTERER, H. Properties and potential of natural pesticides from the neem tree, Azadirachta indica. Annual Review of Entomology, Palo Alto, v. 35, n. 1, p. 271-297, 1990.

SpeciesLINK. 2013. Disponível em: <www.splink.org.br>. Acesso em: set. 2016.

THOMSON, L. A. J.; EVANS, B. Terminalia catappa (tropical almond). In: SPECIES PROFILES FOR PACIFIC ISLAND AGROFORESTRY, 20, 2006. Holualoa: Permanent Agriculture Resources. Disponível em: <www.traditionaltree.org $>$.

TROPICOS - MISSOURI BOTANICAL GARDEN. Tropicos. 2014. Disponível em: <http://www.tropicos.org/>. Acesso em: 23 julho de 2016.

VENZKE, T. S.; NERI, A. V.; CUNHA, J. F.; MARTINS, S. V. Regeneração natural do estrato arbóreo-arbustivo sob talhão de Pinus caribaea var. hondurensis, Viçosa, MG, Brasil. Global Science and Technology, Rio Verde, v. 5, n. 3, p. 74-86, 2012.

WEE, K. L.; WANG, S. Effect of post-harvest treatment on the degradation of mimosine in Leucaena leucocephala leaves. Journal of the Science of Food and Agriculture, Davis, v. 39, n. 3, p. 195 201, 1987.

WILLIAMSON, M. Invasions. Ecography, Lund, v. 22, n. 1, p. 5-12, 1999.

YOSHIDA, K.; OKA, S. Invasion of Leucaena leucocephala and its Effects on the Native Plant Community in the Ogasawara (Bonin) Islands 1. Weed Technology, Lawrence, v. 18, n. sp1, p. 1371-1375, 2004.

ZALBA, S. M. Introdução às invasões biológicas - Conceitos e definições. In: BRAND, K. (Ed.). América do Sul invadida: a crescente ameaça das espécies exóticas invasoras. Nairobi: GISP, 2006. p. 4-14.

ZILLER, S. R.; ZENNI, R. D.; DECHOUM, M. S. Espécies exóticas invasoras na arborização urbana: problemas e soluções. In: CONGRESSO BRASILEIRO DE ARBORIZAÇÃO URBANA, XI, 2007, Vitória. Anais... Vitória, 2007. p. 18.

ZVIEJKOVSKI, I. P.; CAMPOS, J. B.; CAMPOS, R. M.; LANDGRAF, G. O. Potencial invasor de Psidium guajava em um intervalo de cinco anos (2002-2007) dentro de uma Unidade de Conservacão. In: CONGRESSO DE ECOLOGIA DO BRASIL, XI, 2009, São Lourenço. Anais... São Lourenço: SBE, 2009. p. 3. 